\title{
A new heterozygous mutation of the FOXL2 gene is associated with a large ovarian cyst and ovarian dysfunction in an adolescent girl with blepharophimosis/ptosis/epicanthus inversus syndrome
}

\author{
K Raile, H Stobbe, R B Tröbs ${ }^{1}$, W Kiess and R Pfäffle \\ Hospital for Children and Adolescents and ${ }^{1}$ Hospital for Pediatric Surgery, Faculty of Medicine, University of Leipzig, Oststr. 21-25, D-04317 Leipzig, \\ Germany
}

(Correspondence should be addressed to K Raile; Email: klemens.raile@medizin.uni-leipzig.de)

\begin{abstract}
Blepharophimosis/ptosis/epicanthus inversus syndrome (BPES), an autosomal dominant syndrome in which eyelid malformation is associated with (type I BPES) or without premature ovarian failure (type II BPES). Mutations of a putative winged helix/forkhead transcription factor FOXL2 account for both types of BPES. We report on a 16-year-old adolescent girl with blepharophimosis and ptosis. Subsequently she developed oligomenorrhea, secondary amenorrhea for 6 months, and an extremely large cyst of one ovary. The cyst contained 81 of cyst fluid and histopathology displayed a large corpus luteum cyst. Following laparotomy, gonadotropin levels were elevated (LH 17.2 U/l, FSH $29.4 \mathrm{U} / \mathrm{l})$ and estradiol levels decreased ( $67 \mathrm{pmol} / \mathrm{l})$. Because of clinical aspects of BPES and abnormal ovarian function we suspected a mutation of her FOXL2 gene and found a new in-frame mutation (904_939dup36) on one allele, leading to a 12 alanine expansion within the polyalanine domain. We conclude that the FOXL2 mutation 904_939dup36 may account not only for blepharophimosis and ptosis but also for ovarian dysfunction and growth of the large corpus luteum cyst. In contrast to known FOXL2 mutations with polyalanine expansions and association with BPES type II, clinical aspects of our girl may indicate some degree of ovarian dysfunction that might finally lead to BPES type I with premature ovarian failure.
\end{abstract}

European Journal of Endocrinology 153 353-358

\section{Introduction}

Blepharophimosis/ptosis/epicanthus inversus syndrome (BPES; MIM\#110100) is predominantly characterized by malformation of the external eye with blepharophimosis, ptosis and a variable degree of epicanthus inversus. There are two clinical subtypes of this disease characterized by the clinical presence or absence of premature ovarian failure (POF). In females, type I BPES is associated with POF, while type II is not (1). Mutations in FOXL2 (GenBank accession number AF301906), a putative forkhead transcription factor gene located at chromosome $3 \mathrm{q} 23$, has been found to cause both types of BPES (2). Other members of the large family of winged helix/forkhead transcription factors are involved in different developmental and metabolic processes and some of them are responsible for genetic, developmental diseases (3). In mice, Foxl2 mRNA is expressed in embryonic eyelids and in embryonic and adult ovary $(2,4)$. Furthermore, FOXL2 is the first autosomal transcription factor expressed in the female differentiation of the embryonal gonad and the murine Foxl2 gene is essential for differentiation of granulosa cells and maintenance of ovarian structure and function $(4,5)$. Ovaries from homozygous Foxl2 $2^{\text {lacZ }}$ knockout mice show that nearly all primordial follicles have initiated folliculogenesis but functional granulosa cells are absent. This constellation led to early oocyte atresia and progressive follicular depletion (5). Furthermore, FOXL2 was found to repress the promoter activity of the steroidogenic acute regulatory (StAR) gene. Dominant negative mutations of FOXL2 could increase expression of StAR and other follicular differentiation factors (6). Pisarska et al. (6) hypothesized that increased StAR expression results in higher initial recruitment of dormant follicles and thus in the POF phenotype. Whether ovarian malfunction in type I BPES patients is caused by early abnormalities in the development of the fetal ovary or by premature degeneration of the adult ovary, or by both, is still controversial $(2,4)$.

FOXL2 is encoded by a single-exon gene of $12745 \mathrm{~kb}$. The FOXL2 protein of 376 amino acids in length includes a 110 amino acid winged-helix forkhead domain important for DNA binding and an alanine/proline-rich C-terminal domain similar to the 
ones of other homeobox proteins (6). In some forkhead family proteins, these alanine- and proline-rich regions have been credited with repressing transcriptional activity, especially in genes involved in organ differentiation $(7,8)$.

Initially a strong genotype-phenotype correlation was suggested (2), but studying larger cohorts, a strong phenotype variability between mutations within the same nucleotide region and even within family members carrying the same mutation was found. Thus genotype-phenotype correlation cannot be generalized $(9,10)$ and patients carrying FOXL2 mutations challenge genetic counseling (9).

In this study, we report on a 16-year-old adolescent girl with blepharophimosis and ptosis. Subsequently she developed oligomenorrhea, secondary amenorrhea, and finally a large corpus luteum cyst of one ovary. We analyzed her genomic DNA for a mutation of the FOXL2 gene and found a new in-frame mutation, 904_939dup36. The putative FOXL2 protein is elongated and contains a duplication of 12 alanine amino acids within the polyalanine amino acid domain.

\section{Case report and clinical follow-up}

The index patient is a female, who was born at term after an uneventful pregnancy. At birth, she was noted to have a symmetric deformity of her eyelids with blepharophimosis and ptosis. Otherwise, physical examination of the newborn was normal. In detail, she had no microcephaly or other dysmorphic features. Postnatally, the girl developed normal physical and psychomotor skills. She entered puberty appropriately and experienced menarche at the age of 13 years 6 months. Following menarche, menstruation was irregular every 6-8 weeks (oligomenorrhea) and finally stopped at the age of 15 years 2 months (secondary amenorrhea). Thereafter, the girl reported one short episode of abdominal pain and within the following 3 months experienced an otherwise unexplained weight gain of approximately $6 \mathrm{~kg}$. Her abdominal circumference was increasing rapidly, making her feel like being 'pregnant'. On presentation abdominal ultrasound and subsequently a magnetic resonance imaging (MRI) scan of the abdomen displayed a huge, cystic tumor that filled out the entire abdominal cavity (Fig. 1A). The tumor marker proteins beta-human chorionic gonadotropin and alpha-fetoprotein were negative and we suspected a large ovarian cyst. The patient underwent laparotomy and the huge cyst was found to have its origin in the left ovary. The cyst was punctured and removed after approximately $8 \mathrm{l}$ of cystic fluid had been released. The left ovary was macroscopically still intact and thus was not removed. The histology of the cyst wall showed an ovarian tissue with low-grade fibrosis and single primordial follicles. The internal side was covered with luteinized follicular epithelium. Cytology of the cystic
A

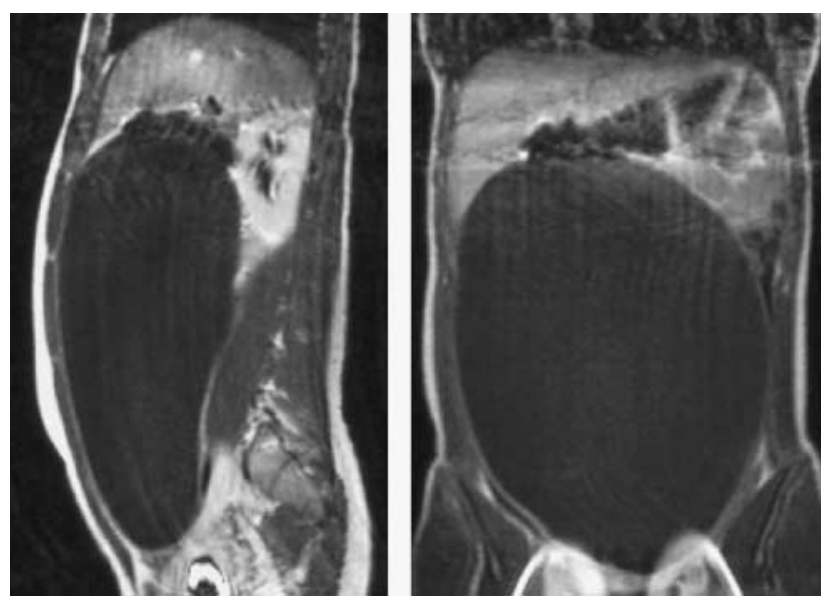

$\mathrm{B}$

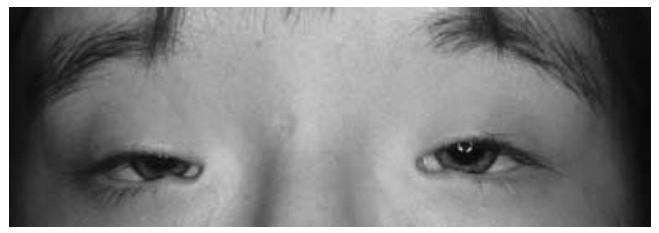

Figure 1 (A) MRI scan of the abdomen. The cystic ovarian tumor contained $8 \mathrm{I}$ of fluid and filled the abdominal cavity. Cytology of the cystic fluid and microscopy of cyst tissue gave evidence for a non-malignant corpus luteum cyst. (B) Eyelid defects in our BPES patient at the age of 16.5 years. A shortening of the horizontal eye fissure (blepharophimosis) and lowering of the vertical, palpebral fissure (ptosis) is evident. The arch-like eyebrows indicate an impairment of the levator palpebrae superioris muscle and compensation by the frontalis muscle.

fluid displayed only luteinized cells, which makes a large corpus luteum cyst the most likely diagnosis.

We saw the adolescent girl 6 weeks after surgery at our endocrine clinic. On examination at the age of 15.5 years height was $175.5 \mathrm{~cm}$ (97th percentile), weight $53.4 \mathrm{~kg}$ (50th percentile) and pubertal Tanner stages PH4 and B4, and a general physical examination normal. We found a prominent blepharophimosis and ptosis on both eyes but no abnormalities in visual function (Fig. 1B).

The combination of the observed eye abnormalities with her signs of ovarian dysfunction (secondary amenorrhea and the corpus luteum cyst) let us to assume BPES and we sequenced her FOXL2 gene. The family history revealed no other cases of eyelid deformities or female cases with POF or infertility; thus we suppose a sporadic mutation in our patient (Fig. 2).

\section{Methods}

Clinical and molecular studies were performed after obtaining written consent from the patient and her parents. 


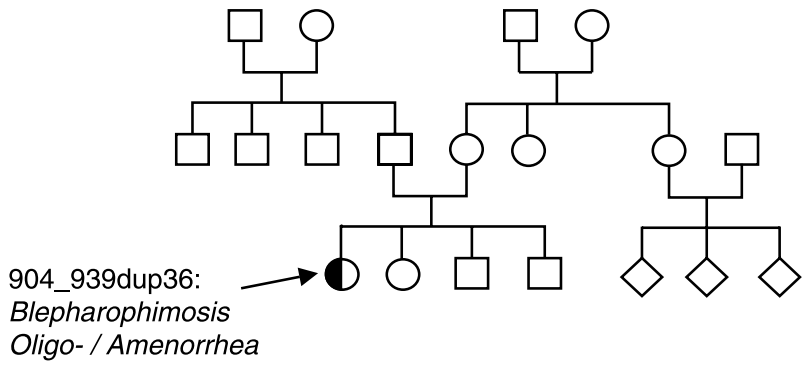

Figure 2 Pedigree of the patient's family. No other family members showed eye deformities or clinical aspects of POF (females). Thus we assume a sporadic mutation in our patient.

\section{Hormone assays}

Serum levels of follicle-stimulating hormone (FSH), luteinizing hormone (LH), estradiol, progesterone, thyrotropin, free triiodothyronine, free thyroxine and insulin-like growth factor (IGF)-I were measured by commercially available assays: Measurement of LH, FSH, estradiol and thyroid hormones was performed by the Elecsys/Modular system (Roche, Mannheim, Germany). IGF-I was determined by the Advantage system (Nichols Institute, Bad Vilbel, Germany). Intraas well as inter-assay coefficients of variation for all methods were below $8 \%$ in the normal range. Agerelated normal values were LH 1.1-7.4 U/l, FSH 3.3$10.3 \mathrm{U} / \mathrm{l}$ and estradiol $67-907 \mathrm{pmol} / \mathrm{l}$.

\section{DNA isolation, amplification and sequencing}

Genomic DNA was isolated from peripheral blood lymphocytes using standard DNA extraction methods. For mutation screening, direct sequencing of the genomic DNA of the human FOXL2 gene was performed. Primers for DNA fragments were used as previously reported by Crisponi et al. (2). We performed PCR reactions in a $25 \mu \mathrm{l}$ volume with $100 \mathrm{ng}$ genomic DNA and the following parameters: $30 \mathrm{~s}$ at $92{ }^{\circ} \mathrm{C}, 30 \mathrm{~s}$ at $56^{\circ} \mathrm{C}$ and $1 \mathrm{~min}$ at $72^{\circ} \mathrm{C}$ for 30 cycles. Sequencing of the FOXL2 DNA was performed using the ABI PRISM BigDye Primer Cycle Sequencing Kit on an ABI Prism 310 DNA sequencer (Applied Biosystems, Weiterstadt, Germany) according to the manufacturer's instructions.

\section{Results}

\section{Genetic analysis}

The DNA fragments of the entire coding region of the human FOXL2 gene were amplified by PCR. The data obtained were compared with the published sequence of the human FOXL2 gene (gene bank accession number \#605597). The patient had a new heterozygous mutation with a $36 \mathrm{bp}$ duplication of the FOXL2 gene at cDNA position 904-939 (904_939 dup36). The duplicated base pairs encode for 12 of a total of
14 alanines of the polyalanine region of the FOXL2 protein. Thus the expected protein transcript should display a duplication and elongation within the polyalanine stretch of the FOXL2 transcript (Fig. 3). No further polymorphisms or mutations were detected on other positions of the FOXL2 gene. We suppose that the patient has a new mutation, based on the fact that no other family member has blepharophimosis. We regret that the parents did not agree to further mutation analysis on them and on other family members, which means that we cannot present complete genetic data on the family.

\section{Serum concentrations of hormones and clinical follow-up}

The patient was followed up for 20 months after ovarian surgery. Before surgery, no hormone levels had been obtained. On day 4 after surgery, estradiol levels were low $(30 \mathrm{pmol} / \mathrm{l})$, gonadotropin levels were not elevated (LH 5.0 U/l, FSH $7.7 \mathrm{U} / \mathrm{l}$ ) and progesterone levels $(0.9 \mathrm{pmol} / \mathrm{l})$ were in agreement with a preovulatory stage. After 6 and 7 weeks, estradiol levels were low (6 weeks: $136.0 \mathrm{pmol} / \mathrm{l}$ and 7 weeks after surgery: $67.0 \mathrm{pmol} / \mathrm{l}$ ) and gonadotropin levels elevated. FSH levels were higher than LH levels after 6 weeks (FSH $14.3 \mathrm{U} / \mathrm{l}$, LH $11.3 \mathrm{U} / \mathrm{l}$ ) and 7 weeks (FSH $29.4 \mathrm{U} / \mathrm{l}$, LH $17.2 \mathrm{U} / \mathrm{l})$ indicating ovarian insufficiency. At this point the development of primary ovarian insufficiency was much more likely than a preovulatory state, because gonadotropins showed a further increase from 6-7 weeks after surgery and progesterone levels remained low (1.1 pmol/l) after 7 weeks (Fig. 4).

Three months after surgery, spontaneous menstrual bleedings started, estradiol levels normalized and gonadotropin levels decreased. After then, menstrual cycles occurred regularly every $4-5$ weeks for the following 17 months, interrupted by one interlude of amenorrhea and elevated gonadotropin levels (FSH 38.0 U/l, LH $22.4 \mathrm{U} / \mathrm{l}$, estradiol $83 \mathrm{pmol} / \mathrm{l}$ ) for 3 months. Thus we have some aspects of ovarian dysfunction but not of terminal ovarian failure.

Thyroid function, IGF-I and prolactin levels were within the age-adjusted normal range. Karyotyping showed a normal female karyotype 46,XX in approximately 100 mitotic lymphocytes. Subsequent ultrasound examinations displayed only small follicular cysts of both ovaries.

\section{Discussion}

In this girl, phenotypic characteristics of BPES in terms of eye malformation were present. Her history of oligomenorrhea and secondary amenorrhea, associated with the growth of an extremely large ovarian cyst suggested ovarian dysfunction and therefore we suspected a FOXL2 mutation. By direct sequencing of 
FOXL2 Wild Type

Forkhead domain Poly-ALA

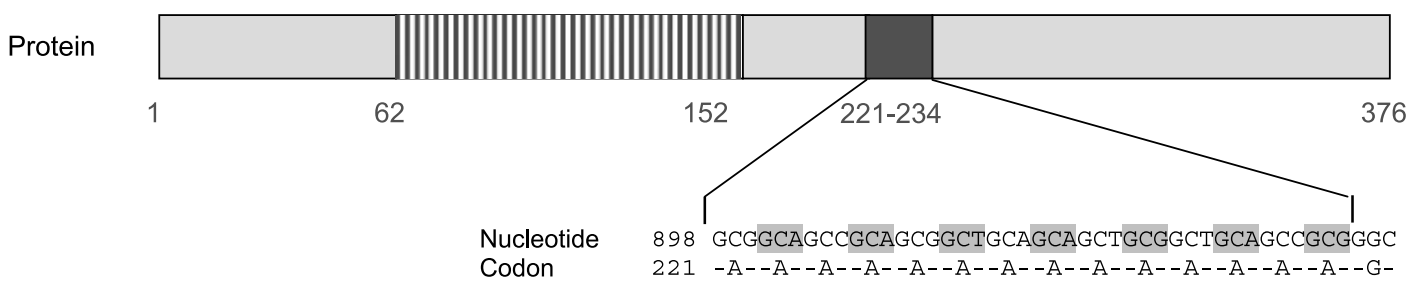

FOXL2 Mutation

904_939dup36

Forkhead domain

Dup Poly-ALA

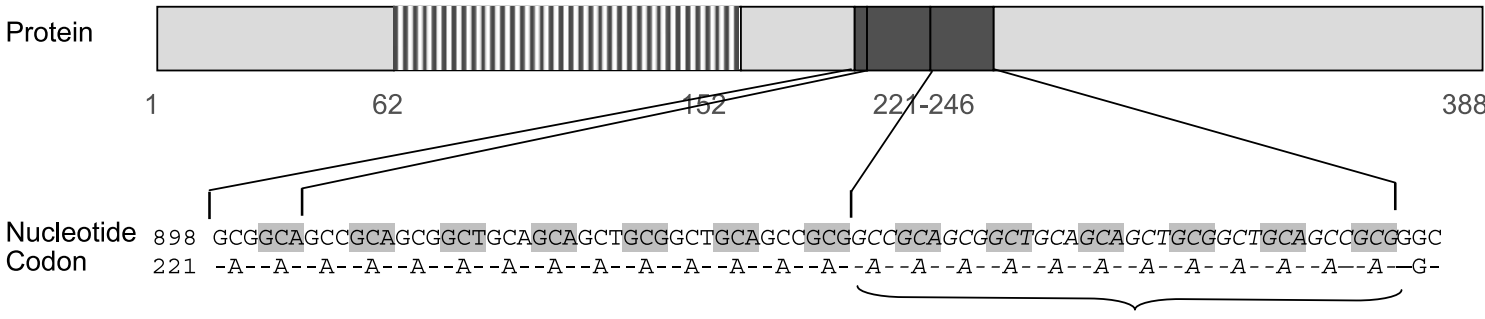

duplicated sequence

Figure 3 Nucleotide sequence, codons and putative protein structure of wild-type and mutant (904_939 dup36) FOXL2. The predicted protein contains a duplication within the polyalanine domain leading to an elongation of the polyalanine stretch from 14 to 26 alanines. Polyalanine stretches form helixes and are hydrophobic protein regions. Elongated hydrophobic polyalanine regions may lead to increased FOXL2 protein aggregation not only of mutant but also of wild-type protein. Thus the amount of free and functional protein may be diminished.

her FOXL2 gene we found a new in-frame mutation (904_939 dup36) of one FOXL2 allele. The putative FOXL2 protein results in a nearly complete duplication of the whole polyalanine region.

\section{FOXL2 mutation, primary ovarian failure and growth of the corpus luteum cyst}

Large ovarian cysts of the size we reported here are extremely uncommon. Single cases have been reported during pubertal development or pregnancy, or in premenopausal women. During puberty and menopause, high gonadotropin levels reflect either incomplete ovarian function of the developing (puberty) or degenerating (premenopausal) ovary. During pregnancy, high levels of placental gonadotropin levels are thought to stimulate cyst growth $(11,12)$. Furthermore, luteinized Graafian follicles have been reported to develop in women with POF and inappropriate luteinization of follicles appears to be a major pathophysiological mechanism in patients with karyotypically normal spontaneous POF (13). In our girl we found histopathology of a large corpus luteum cyst and a hormonal constellation of transient ovarian insufficiency. Malfunction of FOXL2 protein may have contributed to primary ovarian insufficiency, followed by high gonadotropin levels and stimulation of a corpus luteum cyst. In addition, a luteinized Graafian follicle may have shown abnormal response to these increased LH and FSH levels. So far FOXL2 mutations have been reported that were associated either with delayed puberty and a hormonal constellation of primary ovarian failure $(737 \mathrm{~T}>\mathrm{A}, 738 \mathrm{C}>\mathrm{A}(2))$, or with a very late menarche at adult age, combined with POF before the age of 35 years (989_996del (2)).

Our girl is the first reported case who presented with abnormal growth of a large ovarian cyst. The phenotype of homozygous Foxl2 knockout mice indicates that complete Foxl2 deactivation leads to fewer functional granulosa cells, premature oocyte maturation, early oocyte depletion and finally to early degeneration of the ovary (5). Furthermore, FOXL2 was found to repress StAR expression, and like other FKH/HNF3 family members might repress other ovarian genes involved in the differentiation of the ovary (6-8). Human FOXL2 might regulate differentiation and growth during embryonal development and ensure adult ovarian functions, like follicle growth, ovulation and corpus luteum regression. FOXL2 mutation therefore could account for the various aspects of ovarian dysfunction observed in our case and in type I BPES patients.

\section{Phenotype in BPES subjects and FOXL2 sequence and structure}

FOXL2 contains a characteristic 110 amino acid DNAbinding domain, the forkhead, and a 14 amino acid polyalanine stretch downstream of the forkhead 

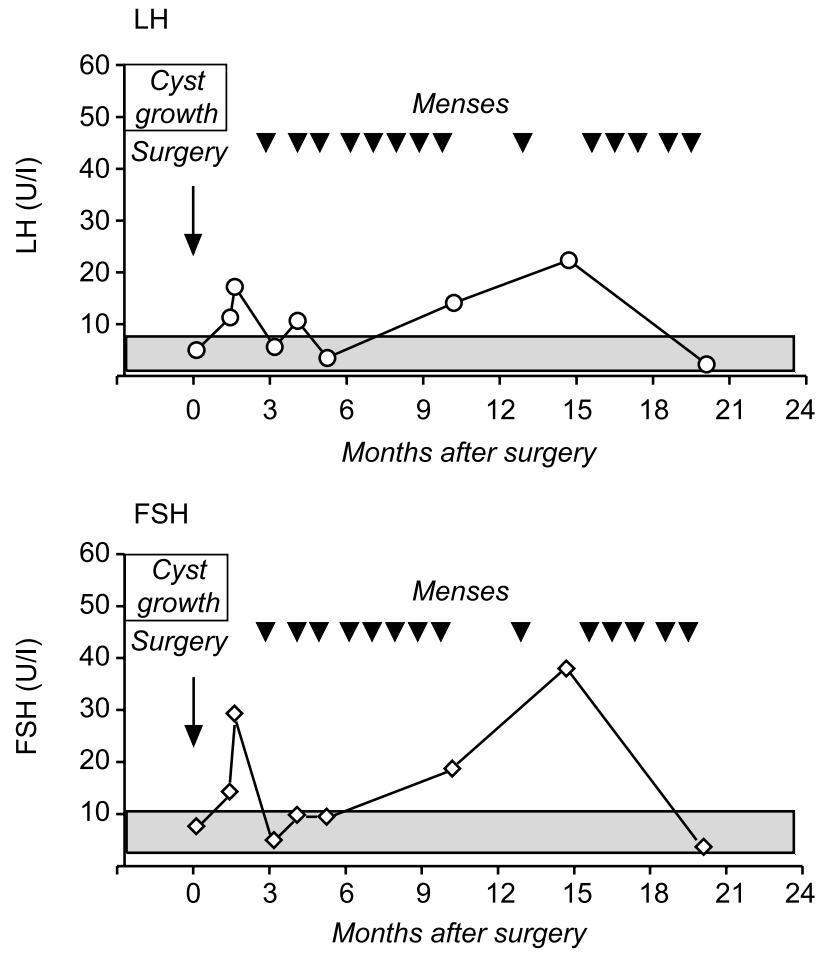

$\mathrm{E}_{2}$

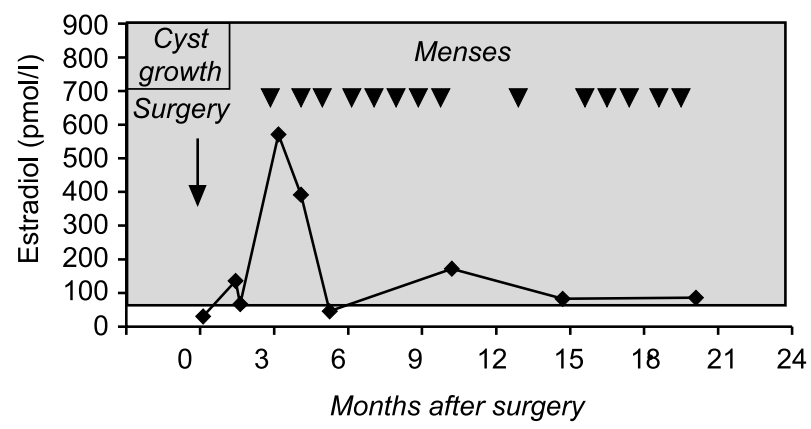

Figure 4 Basal serum LH and FSH levels (upper panels) as well as serum estradiol $\left(E_{2}\right)$ levels (lower panel) after surgical removal of the large corpus luteum cyst. Three months after surgery spontaneous menstruation started (triangles). Grey areas are agerelated normal control values (LH 1.1-7.4 U/l, FSH 3.3-10.3 U/l, estradiol 67-907 pmol/l).

domain (2). Human FOXL2 mutations leading to truncated proteins without a forkhead domain or with a complete forkhead domain but with loss of the polyalanine stretch result almost certainly in BPES type I with ovarian failure $(14,15)$. The in-frame mutation in our patient, 904_939 dup36, results in a large duplication of 12 alanine amino acids and leads to an elongated polyalanine stretch with 26 alanine amino acids instead of 14. Reported in-frame mutations leading to polyalanine extensions are 909_911delAGC;912_938dup27 (2), 909_938dup30 (2, 14-16), 900_929dup30 (14, 15) and 921_935trip15 (15). The phenotypes of female patients with these mutations were either unknown or BPES type II. Only in the family with the 909_911delAGC;912_938dup27 mutation had fertility problems and premature child birth been reported in two affected females $(2,15)$. So far, De Baere et al. (15) conclude that patients carrying polyalanine expansions most likely show BPES type II, with most reported females being fertile. In addition to these known FOXL2 mutations with polyalanine extensions, the mutation reported here is associated with oligomenorrhea, intermittent secondary amenorrhea and growth of a large corpus luteum cyst, indicating some degree of ovarian dysfunction. But after laparotomy and cyst removal, the drop in gonadotropin levels, increase of serum estradiol and a return of regular menstrual cycles would indicate at least a partial regain of normal ovarian function. Nevertheless, temporary restoration of normal ovarian function has been reported in women with POF (17), and at this point we cannot predict whether the observed symptoms may lead to definitive ovarian failure in our girl. Criteria for POF in BPES type I patients were defined as cessation of menses for a duration of $>6$ months at an age $<40$ years and an FSH concentration $>40 \mathrm{U} / \mathrm{l}$ (15). Until now, our patient has not met these well-defined criteria. Finally, genotype-phenotype correlation in subjects with FOXL2 mutations leading to polyalanine extensions is not as clear cut as would be expected and genetic counseling should be considered carefully (9).

\section{Structure of the FOXL2 904_939 dup36 mutant protein and functional aspects}

FOXL2 protein structure is conserved through several vertebrate species (18). While the forkhead domain is highly conserved in all vertebrate species, in mammals the polyalanine tract is restricted to a maximal length of 14 alanine residues, while in fish it is completely missing (19). This fact suggests that the length of the polyalanine tract might tune transcriptional factor activity of FOXL2 with 14 alanines being the optimal length for FOXL2 function in mammals $(18,19)$. However, mutant FOXL2 protein with expansions of the polyalanine tract from 14 to 24 residues was found to form intranuclear and cytoplasmic aggregates, and co-transfection experiments suggest that wild-type and mutant proteins can co-aggregate. Thus the molecular pathogenesis of FOXL2 mutants with polyalanine extensions might be a mislocation of mutant and wild-type protein with its inclusion into nuclear aggregates. This may diminish the pool of active, functional FOXL2 protein (20). Molecular dysfunction of our FOXL2 mutation with a polyalanine expansion from 14 to 26 residues may be in this way, although we found a rare ovarian dysfunction in our case that has not been reported for the very frequent mutations 909_938dup30 (2, 14-16) and 900_929dup30 (14, 15). Moreover, functional studies in polyglutamine repeat (polyQ) diseases, including Huntington disease, Kennedy disease or 
spinocerebellar ataxia, showed for the respective mutant proteins unstable expansions beyond the normal range, with longer expansions correlating with an earlier onset and more severe phenotype of disease (21). In vitro, mutant proteins with expanded polyglutamine stretches form ubiquitinated intraneuronal nuclear inclusion bodies caused by abnormal protein folding (22). If these experiences in polyQ diseases were applied to FOXL2 mutations with polyalanine expansions, the length of polyalanine extensions should correlate with ovarian malfunction. Whether this is true remains to be investigated.

We conclude that this new FOXL2 in-frame mutation, 904_939 dup36, might account not only for the blepharophimosis and ptosis but also for ovarian dysfunction and growth of a large corpus luteum cyst. The FOXL2 mutation with an elongated polyalanine domain that we report here is associated with at least a temporary impairment of ovarian function and may lead to POF, as observed in patients with BPES I. Whereas blepharophimosis seems a constant feature of FOXL2 mutations, occurrence and onset of POF are more variable. In the face of this, genetic counseling of patients with FOXL2 mutations remains difficult.

\section{Acknowledgements}

We acknowledge the contribution of Torsten Schöneberg, Department of Biochemistry, Faculty of Medicine, University of Leipzig, for discussing protein structure and potential impact on function of the mutated protein.

\section{References}

1 Zlotogora J, Sagi M \& Cohen T. The blepharophimosis, ptosis, and epicanthus inversus syndrome: delineation of two types. American Journal of Human Genetics 198335 1020-1027.

2 Crisponi L, Deiana M, Loi A, Chiappe F, Uda M, Amati P, Bisceglia L, Zelante L, Nagaraja R, Porcu S, Ristaldi MS, Marzella R, Rocchi M, Nicolino M, Lienhardt-Roussie A, Nivelon A, Verloes A, Schlessinger D, Gasparini P, Bonneau D, Cao A \& Pilia G. The putative forkhead transcription factor FOXL2 is mutated in blepharophimosis/ptosis/epicanthus inversus syndrome. Nature Genetics 200127 159-166.

3 Carlsson P \& Mahlapuu M. Forkhead transcription factors: key players in development and metabolism. Developmental Biology $20022501-23$.

4 Loffler KA, Zarkower D \& Koopman P. Etiology of ovarian failure in blepharophimosis ptosis epicanthus inversus syndrome: FOXL2 is a conserved, early-acting gene in vertebrate ovarian development. Endocrinology 2003144 3237-3243.

5 Schmidt D, Ovitt CE, Anlag K, Fehsenfeld S, Gredsted L, Treier AC \& Treier M. The murine winged-helix transcription factor Foxl2 is required for granulosa cell differentiation and ovary maintenance. Development $2004131933-942$.

6 Pisarska MD, Bae J, Klein C \& Hsueh AJW. Forkhead L2 is expressed in the ovary and represses the promoter activity of the steroidogenic acute regulatory gene. Endocrinology 2004 $1453424-3433$.

7 Sullivan SA, Akers L \& Moody SA. FoxD5a, a Xenopus winged helix gene, maintains an immature neural ectoderm via transcriptional repression that is dependent on the C-terminal domain. Developmental Biology 2001232 439-457.

8 Sutton J, Costa R, Klug M, Field L, Xu D, Largaespada DA, Fletcher CF, Jenkins NA, Copeland NG, Klemsz M \& Hromas R. Genesis, a winged helix transcriptional repressor with expression restricted to embryonic stem cells. Journal of Biological Chemistry $199627123126-23133$.

9 Fokstuen S, Antonarakis SE \& Blouin JL. FOXL2-mutations in blepharophimosis-ptosis-epicanthus inversus syndrome (BPES): Challenges for genetic counseling in female patients. American Journal of Medical Genetics 2003117 143-146.

10 Udar N, Yellore V, Chalukya M, Yelchits S, Silva-Garcia R \& Small K. Comparative analysis of the FOXL2 gene and characterization of mutations in BPES patients. Human Mutation 200322 222-228.

11 Clement PB \& Scully RE. Large solitary luteinized follicle cyst of pregnancy and puerperium: A clinicopathological analysis of eight cases. American Journal of Surgical Pathology 19804 431-438.

12 Haddad A, Mulvany N, Billson V \& Arnstein M. Solitary luteinized follicle cyst of pregnancy. Report of a case with cytologic findings. Acta Cytologica $2000 \mathbf{4 4} 454-458$.

13 Nelson LM, Anasti JN, Kimzey LM, Defensor RA, Lipetz KJ, White BJ, Shawker TH \& Merino MJ. Development of luteinized Graafian follicles in patients with karyotypically normal spontaneous premature ovarian failure. Journal of Clinical Endocrinology and Metabolism 199479 1470-1475.

14 De Baere E, Dixon MJ, Small KW, Jabs EW, Leroy BP, Devriendt K, Gillerot Y, Mortier G, Meire F, Van Maldergem L, Courtens W, Hjalgrim H, Huang S, Liebaers I, Van Regemorter N, Touraine P, Praphanphoj V, Verloes A, Udar N, Yellore V, Chalukya M, Yelchits S, De Paepe A, Kuttenn F, Fellous M, Veitia R \& Messiaen L. Spectrum of FOXL2 gene mutations in blepharophimosis-ptosis-epicanthus inversus (BPES) families demonstrates a genotype-phenotype correlation. Human Molecular Genetics $2001101591-1600$.

15 De Baere E, Beysen D, Oley C, Lorenz B, Cocquet J, De Sutter P, Devriendt K, Dixon M, Fellous M, Fryns JP, Garza A, Jonsrud C, Koivisto PA, Krause A, Leroy BP, Meire F, Plomp A, Van Maldergem L, De Paepe A, Veitia R \& Messiaen L. FOXL2 PES: Mutational hotspots, phenotypic variability, and revision of the genotype-phenotype correlation. American Journal of Human Genetics $2003 \mathbf{7 2} 478-487$.

16 Ramirez-Castro JL, Pineda-Trujillo N, Valencia AV, Muneton CM, Botero O, Trujillo O, Vasquez G, Mora BE, Durango N, Bedoya G \& Ruiz-Linares A. Mutations in FOXL2 underlying BPES (Types 1 and 2) in Colombian families. American Journal of Medical Genetics 2002113 47-51.

17 Rebar RW \& Connolly HV. Clinical features of young women with hypergonadotropic amenorrhea. Fertility and Sterility 199053 $804-810$.

18 Cocquet J, De Baere E, Gareil M, Pannetier M, Xia X, Fellous M \& Veitia RA. Structure, evolution and expression of the FOXL2 transcription unit. Cytogenetic and Genome Research 2003101 206-211.

19 Cocquet J, De Baere E, Caburet S \& Veitia RA. Compositional biases and polyalanine runs in humans. Genetics 2003165 1613-1617.

20 Caburet S, Demarez A, Moumne L, Fellous M, De Baere E \& Veitia RA. A recurrent polyalanine expansion in the transcription factor FOXL2 induces extensive nuclear and cytoplasmic protein aggregation. Journal of Medical Genetics 2004 41 932-936.

21 Forman MS, Trojanowski JQ \& Lee VM. Neurodegenerative diseases: a decade of discoveries paves the way for therapeutic breakthroughs. Nature Medicine 200410 1055-1063.

22 Skinner PJ, Koshy BT, Cummings CJ, Klement IA, Helin K, Servadio A, Zoghbi HY \& Orr HT. Ataxin-1 with an expanded glutamine tract alters nuclear matrix-associated structures. Nature $1997389971-974$.

Received 8 April 2005

Accepted 27 May 2005 